\title{
The lattice gluon propagator in Landau gauge at zero and finite temperature
}

\section{Paulo J. Silva*}

CFC, Departamento de Física, Faculdade de Ciências e Tecnologia, Universidade de Coimbra, 3004-516 Coimbra, Portugal

E-mail: psilva@teor.fis.uc.pt

\section{Orlando Oliveira}

CFC, Departamento de Física, Faculdade de Ciências e Tecnologia, Universidade de Coimbra, 3004-516 Coimbra, Portugal

E-mail: orlandodteor.fis.uc.pt

\begin{abstract}
The interplay between the finite volume and finite lattice spacing is investigated using lattice QCD simulations to compute the Landau gauge gluon propagator at zero temperature. Comparing several ensembles with different lattice spacings and physical volumes, we conclude that the dominant effects, in the infrared region, are associated with the use of a finite lattice spacing. The simulations show that decreasing the lattice spacing, while keeping the same physical volume, leads to an enhancement of the infrared gluon propagator. Moreover, we also present results for the Landau gauge gluon propagator at finite temperature.
\end{abstract}

Xth Quark Confinement and the Hadron Spectrum

8-12 October 2012

TUM Campus Garching, Munich, Germany

\footnotetext{
*Speaker.
} 


\section{The gluon propagator at zero temperature}

In [1], we have studied the lattice Landau gauge gluon propagator, using several ensembles with different lattice volumes and lattice spacings. Here we will report on the comparison of the gluon propagator computed from similar physical volumes but different lattice spacings. For details on the lattice setup see [1].

Figure 1 shows the gluon propagator, renormalized at $4 \mathrm{GeV}$, for physical volumes ranging from $3.3 \mathrm{fm}$ to $8.1 \mathrm{fm}$. In all cases, for momenta above $\sim 900 \mathrm{MeV}$ the lattice data define a unique curve; this means that the renormalization procedure has been able to remove all dependence on the ultraviolet cutoff for the mid and high momentum regions.

However, we observe in the infrared region sizeable effects due to the finite lattice spacing of the simulation. In particular, the plots show that large lattice spacing simulations underestimate the propagator in the infrared region. Moreover, we found that the corrections due to the finite lattice spacing are larger than the corrections due to the finite volume of the lattice simulation for details see [1].

One can ask whether the analysis reported here depends on the renormalization scale. Usually, one chooses a renormalization point $\mu$ in the high momentum region, in order to allow a contact with the perturbative behaviour. However, choosing a renormalization point in the infrared is also possible. Here we consider two alternative renormalization points, $\mu=500 \mathrm{MeV}$ and $\mu=1 \mathrm{GeV}$ and study how the propagators differ. Note that we only show results for the simulations performed at $L a \approx 8 \mathrm{fm}$.

Figures 2 and 3 show that for both renormalization points, the differences in the infrared
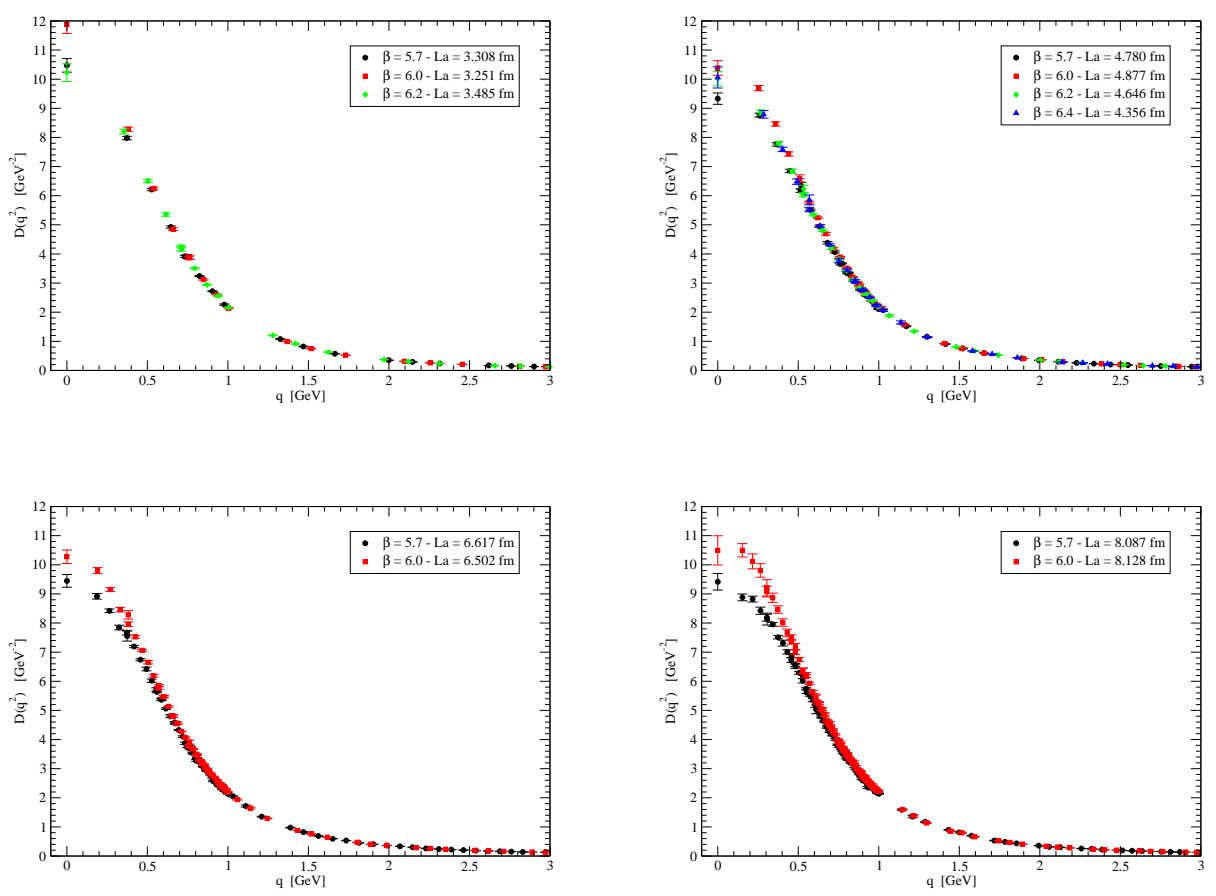

Figure 1: Comparing the renormalized gluon propagator at $\mu=4 \mathrm{GeV}$ for various lattice spacings and similar physical volumes. 

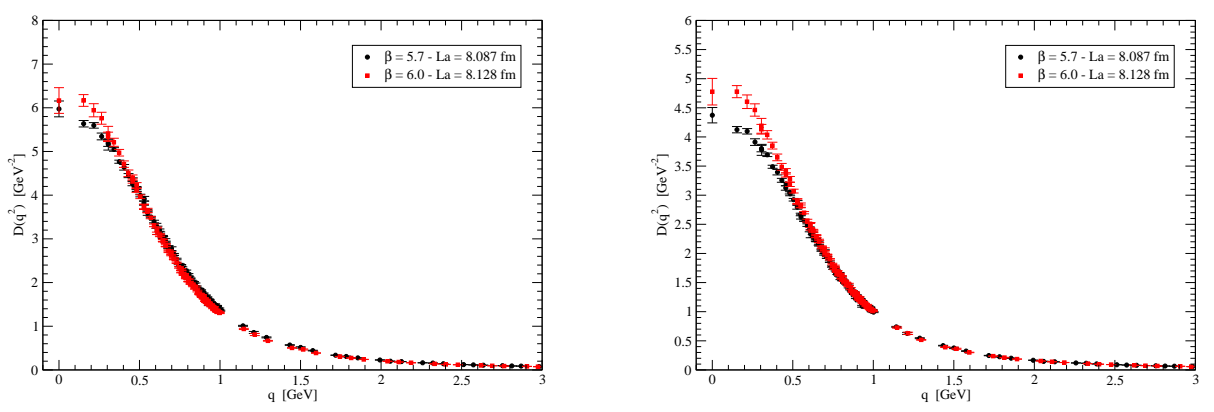

Figure 2: Gluon propagator renormalised at $\mu=500 \mathrm{MeV}$ (left) and at $\mu=1 \mathrm{GeV}$ (right).

region are well beyond one standard deviation. This means that the dominant effect in the infrared propagator is indeed due to the finite lattice spacing, and not the physical box size of the simulation; this conclusion is independent of the renormalization scale. Therefore lattice artifacts due to a finite lattice spacing play an important role in the determination of the infrared behaviour of the gluon propagator in the Landau gauge.
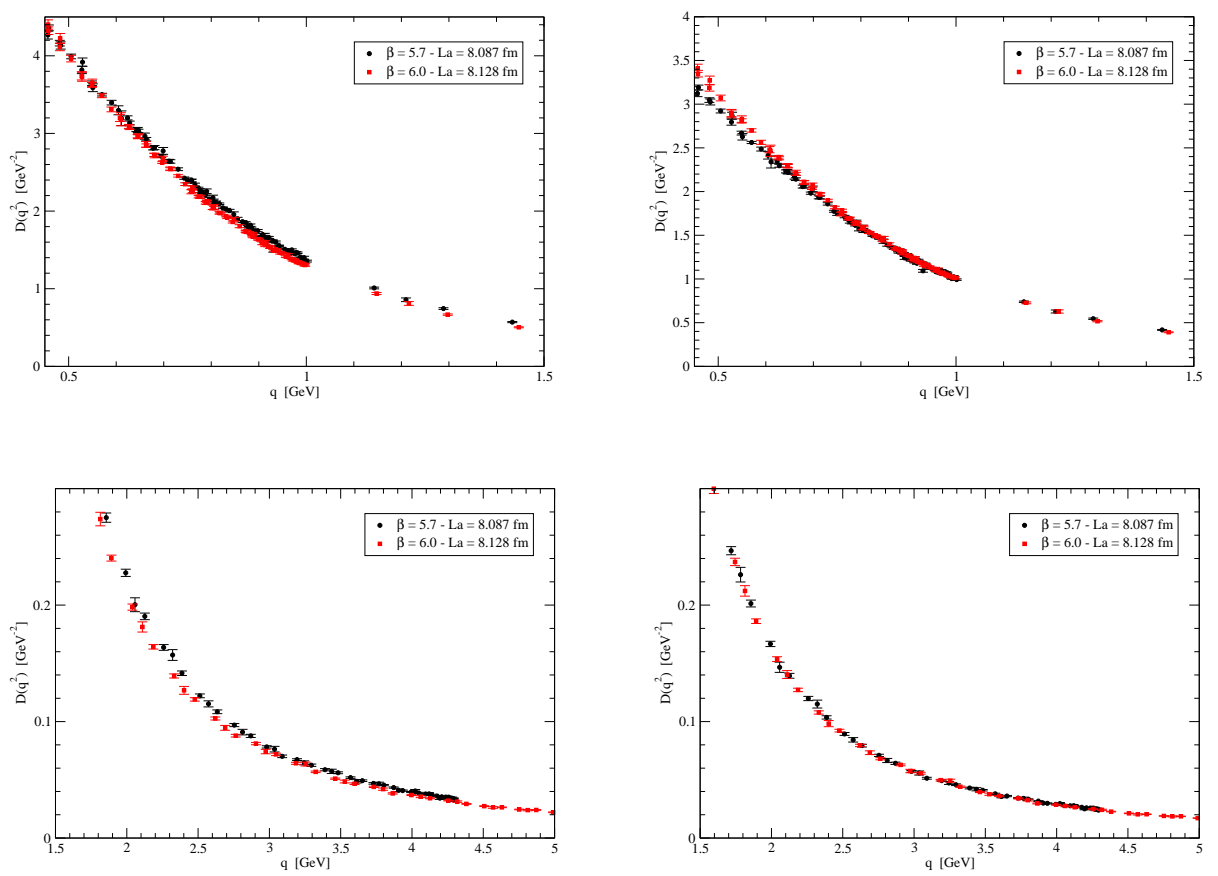

Figure 3: The gluon propagator renormalised at $\mu=500 \mathrm{MeV}$ (left) and at $\mu=1 \mathrm{GeV}$ (right) at intermediate momentum range (top) and up to $5 \mathrm{GeV}$ (bottom). 


\section{The gluon propagator at finite temperature}

Besides the Landau gauge gluon propagator at zero temperature, we are also engaged in the computation of the gluon propagator at finite temperature, in order to understand how temperature changes the gluon propagator $[2,3]$.

Finite temperature is introduced on the lattice through the reduction of the temporal lattice size: the simulations are done on lattices $L_{s}^{3} \times L_{t}$, with $L_{t} \ll L_{s}$. The temperature is defined by $T=1 / a L_{t}$.

At finite temperature, the gluon propagator is described by two tensor structures,

$$
D_{\mu \nu}^{a b}(q)=\delta^{a b}\left(P_{\mu \nu}^{T} D_{T}\left(q_{4}, \vec{q}\right)+P_{\mu \nu}^{L} D_{L}\left(q_{4}, \vec{q}\right)\right)
$$

where the transverse and longitudinal projectors are defined by

$$
P_{\mu v}^{T}=\left(1-\delta_{\mu 4}\right)\left(1-\delta_{v 4}\right)\left(\delta_{\mu v}-\frac{q_{\mu} q_{v}}{\vec{q}^{2}}\right) \quad, \quad P_{\mu v}^{L}=\left(\delta_{\mu v}-\frac{q_{\mu} q_{v}}{q^{2}}\right)-P_{\mu v}^{T}
$$

the transverse $D_{T}$ and longitudinal $D_{L}$ propagators are given by

$$
\begin{gathered}
D_{T}(q)=\frac{1}{2 V\left(N_{c}^{2}-1\right)}\left(\left\langle A_{i}^{a}(q) A_{i}^{a}(-q)\right\rangle-\frac{q_{4}^{2}}{\vec{q}^{2}}\left\langle A_{4}^{a}(q) A_{4}^{a}(-q)\right\rangle\right) \\
D_{L}(q)=\frac{1}{V\left(N_{c}^{2}-1\right)}\left(1+\frac{q_{4}^{2}}{\vec{q}^{2}}\left\langle A_{4}^{a}(q) A_{4}^{a}(-q)\right\rangle\right)
\end{gathered}
$$

The lattice setup is shown in table 2. The finite temperature simulations described in this section have been performed with the help of Chroma library [4]; the FFT transforms have been done with the PFFT library [5]. For the determination of the lattice spacing we fit the string tension data in [6], using the functional form used in [7], in order to have a function $a(\beta)$; the fit has a $\chi^{2} /$ dof $\sim 0.03$.

\begin{tabular}{cccccc}
\hline Temp. $(\mathrm{MeV})$ & $\beta$ & \multicolumn{1}{c}{$L_{s}$} & $L_{t}$ & $\mathrm{a}[\mathrm{fm}]$ & $1 / \mathrm{a}(\mathrm{GeV})$ \\
\hline 121 & 6.0000 & 32,64 & 16 & 0.1016 & 1.9426 \\
162 & 6.0000 & 32,64 & 12 & 0.1016 & 1.9426 \\
243 & 6.0000 & 32,64 & 8 & 0.1016 & 1.9426 \\
260 & 6.0347 & 68 & 8 & 0.09502 & 2.0767 \\
265 & 5.8876 & 52 & 6 & 0.1243 & 1.5881 \\
275 & 6.0684 & 72 & 8 & 0.08974 & 2.1989 \\
285 & 5.9266 & 56 & 6 & 0.1154 & 1.7103 \\
290 & 6.1009 & 76 & 8 & 0.08502 & 2.3211 \\
305 & 5.9640 & 60 & 6 & 0.1077 & 1.8324 \\
305 & 6.1326 & 80 & 8 & 0.08077 & 2.4432 \\
324 & 6.0000 & 32,64 & 6 & 0.1016 & 1.9426 \\
486 & 6.0000 & 32,64 & 4 & 0.1016 & 1.9426 \\
\hline
\end{tabular}

Table 1: Lattice setup used for the computation of the gluon propagator at finite temperature. 

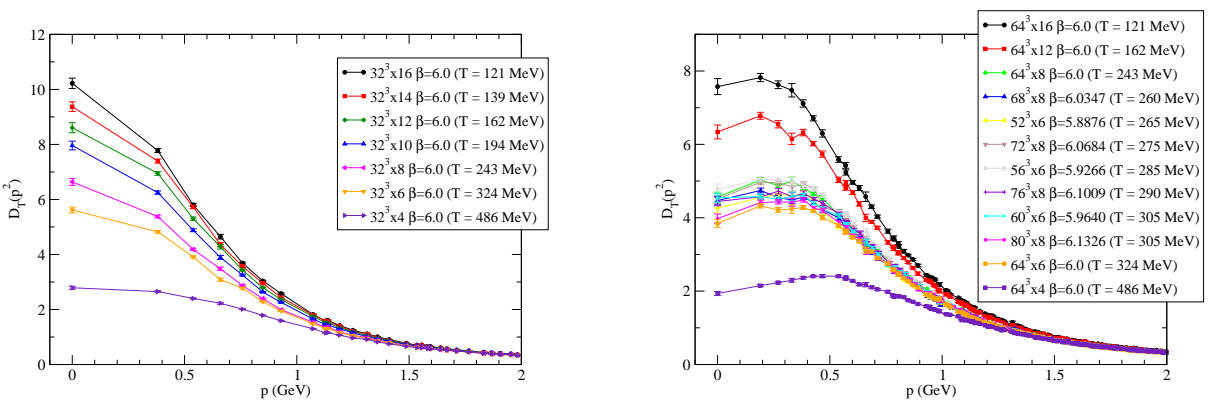

Figure 4: Transverse gluon propagator for $\sim(3.3 \mathrm{fm})^{3}$ (left) and $\sim(6.5 \mathrm{fm})^{3}$ (right) spatial lattice volumes.

The reader should note that the simulation parameters have been carefully chosen, such that we only consider two different spatial physical volumes: $\sim(3.3 \mathrm{fm})^{3}$ and $\sim(6.5 \mathrm{fm})^{3}$. This allows for a better control of finite size effects.

In figures 4 and 5 we show our results. In what concerns the transverse propagator, it decreases with the temperature for low momenta. Moreover, this component shows finite volume effects; in particular, the large volume data exhibits a turnover in the infrared, not seen at the small volume data. The longitudinal component increases for temperatures below $T_{c} \sim 270 \mathrm{MeV}$. Around $T_{c}$, there is a discontinuity and the propagator decreases for $T>T_{c}$. The behaviour of the gluon propagator as a function of the temperature can also be seen in the $3 \mathrm{~d}$ plots shown in figure 6 .
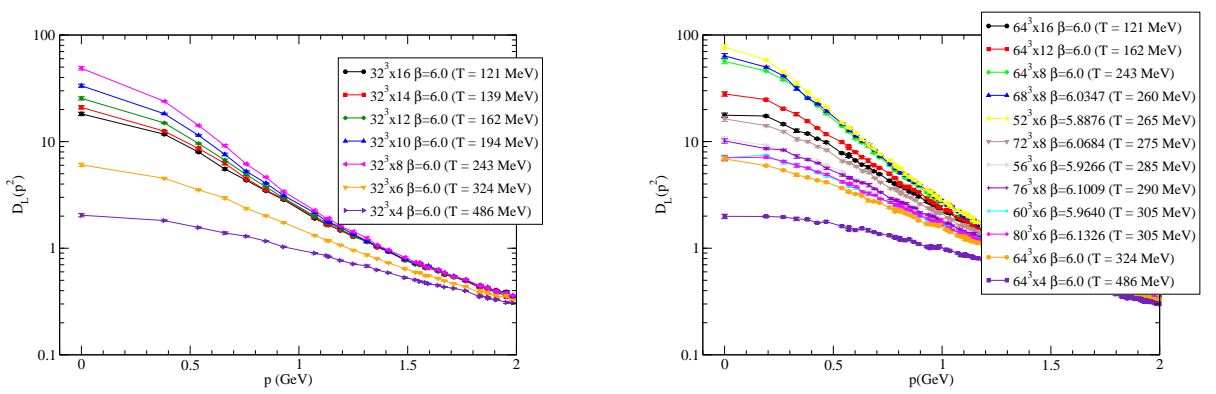

Figure 5: Longitudinal gluon propagator for $\sim(3.3 \mathrm{fm})^{3}$ (left) and $\sim(6.5 \mathrm{fm})^{3}$ (right) spatial lattice volumes.
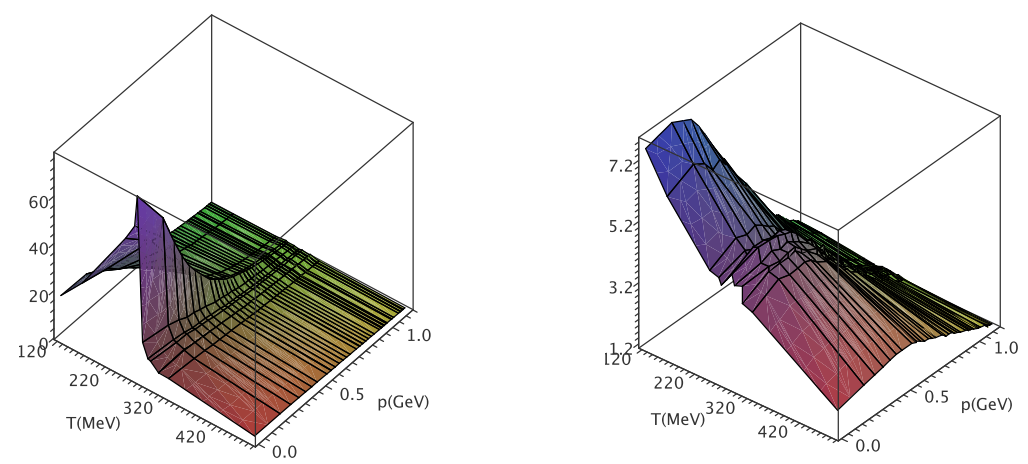

Figure 6: Longitudinal (left) and transverse (right) gluon propagator as a function of momentum and temperature for $\mathrm{a} \sim(6.5 \mathrm{fm})^{3}$ spatial lattice volume. 

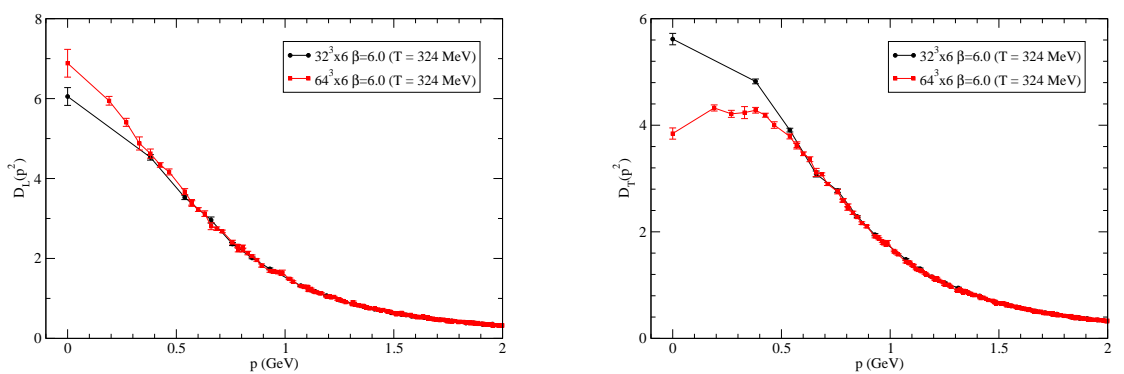

Figure 7: Longitudinal (left) and transverse (right) gluon propagator for different spatial lattice volumes at $\mathrm{T}=324 \mathrm{MeV}$.

The reported finite volume effects can be seen in more detail in figure 7 for a temperature of $324 \mathrm{MeV}$.

A complete analysis should include a study of finite lattice spacing effects. For such a goal, we worked out two different simulations, at $\mathrm{T}=305 \mathrm{MeV}$, with similar physical volumes but different lattice spacings. Figure 8 shows that, for this temperature, finite lattice spacing effects seem to be under control, with the exception of the zero momentum for the transverse component.
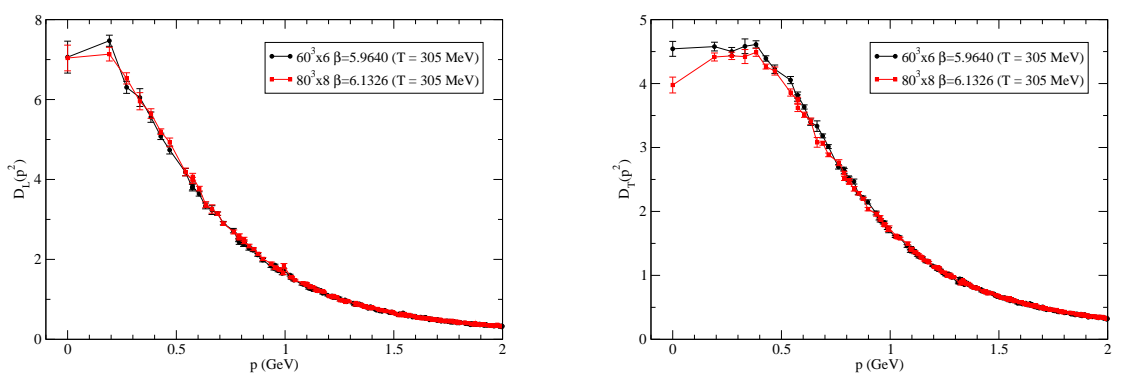

Figure 8: Longitudinal (left) and transverse (right) gluon propagator for different lattice spacings (but similar physical volume) at $\mathrm{T}=305 \mathrm{MeV}$.

Our preliminary results at finite temperature are rather similar to previous works - see for example [8] and references therein. We are currently working on a complete analysis of our data, towards a better understanding of lattice effects on the gluon propagator at finite temperature.

\section{Acknowledgements}

Paulo Silva acknowledges support by FCT under contract SFRH/BPD/40998/2007. Work supported by projects CERN/FP/123612/2011, CERN/FP/123620/2011 and PTDC/FIS/100968/2008, projects developed under initiative QREN financed by UE/FEDER through Programme COMPETE.

\section{References}

[1] O. Oliveira, P. J. Silva, Phys.Rev. D86 (2012) 114513 [arXiv: 1207 . 3029].

[2] O. Oliveira, P. J. Silva, Acta Phys.Polon.Supp. 5 (2012) 1039 [arXiv: 1207 . 7324 ]. 
[3] O. Oliveira, P. J. Silva, PoS (Lattice 2012) 216, [arXiv:1210.8033].

[4] R. G. Edwards, B. Joo, Nucl. Phys. Proc. Suppl. 140 (2005) 832 [arXiv : hep-lat/ 0409003 ].

[5] M. Pippig, PFFT - An Extension of FFTW to Massively Parallel Architectures, Chemnitz University of Technology, 09107 Chemnitz, Germany Preprint 06.

[6] G. Bali, K. Schilling, Phys. Rev. D47 (1993) 661 [arXiv: hep-lat/9208028].

[7] S. Necco, R. Sommer, Nucl.Phys. B622 (2002) 328 [arXiv: hep-lat/ 0108008 ].

[8] A. Cucchieri, D. Dudal, T. Mendes, N. Vandersickel, PoS (QCD-TNT-II) 030 [arXiv:1202.0639]. 